\title{
Full counting statistics of a general quantum mechanical variable
}

\author{
Yu. V. Nazarov \\ Department of Applied Physics and Delft Institute of Microelectronics and Submicrontechnology, \\ Delft University of Technology, Lorentzweg 1, 2628 CJ Delft, The Netherlands \\ M. Kindermann \\ Instituut-Lorentz, Universiteit Leiden, P. O. Box 9506, 2300 RA Leiden, The Netherlands
}

(July 2001)

\begin{abstract}
We present here a quantum mechanical framework for defining the statistics of measurements of $\int d t \hat{A}(t), A(t)$ being a quantum mechanical variable. This is a generalization of the so-called full counting statistics proposed earlier for DC electric currents.

We develop an influence functional formalism that allows us to study the quantum system along with the measuring device thus fully accounting for the action of the detector on the system to be measured. We define the full counting statistics of an arbitrary variable by means of an evolution operator that relates initial and final density matrices of the measuring device.

In this way we are able to resolve inconsistencies that occur in earlier definitions. We suggest two schemes whereby the so defined full statistics can be observed experimentally.

PACS numbers:73.50.Td, 72.70.+m, 73.23.-b,74.40.+k
\end{abstract}

\section{INTRODUCTION}

The measurement paradigm in quantum mechanics assumes at least implicitly that the measurement is instant. 1 . This is to be contrasted with a realistic measurement of, say, electric current where the result of measurement is averaged over a sufficiently long time interval. If one intends to measure a variable $A$, the individual measurement gives $\int_{0}^{T} A(t) d t / T$. The reason for this is obvious: any measurement has to be accurate. The integration over time reduces possible instant fluctuations of $A(t)$ resulting in a more accurate outcome of an individual measurement of this sort. The dispersion of the probability distribution of the outcomes is supposed to vanish in the limit of $T \rightarrow \infty$. This paper focuses on the problems related to the determination of this probability distribution, the full statistics of the measurement results.

Several years ago Levitov and Lesovik 1 日 have made a significant step in the understanding of this fundamental issue. They have introduced a concept of full counting statistics (FCS) of electric current and have found this statistics for the generic case of a one-mode mesoscopic conductor. The word "counting" reflects the discreteness of the electric charge. If electrons were classical particles, one could just count electrons traversing a conductor. The full counting statistics could be readily defined in terms of a probability to have $N$ electrons transferred through the conductor during a time interval $T, P_{T}(N)$. With this distribution function one calculates the average current $\langle N\rangle / T$, current noise $\left(\langle N\rangle^{2}>-\langle N\rangle^{2}\right) / T$ and all higher cumulants of the current. A non-trivial value of interest is the probability to have big deviations from the average value. This can be measured with a threshold detector. The probability distribution $P_{T}(N)$ would be the goal of a quantummechanical calculation.

The operator of electric current through a conductor, $\hat{I}$, is well-defined in the Fock space spanned by the scattering states of electrons. The initial idea of Lesovik and Levitov was to define an operator of transferred charge by means of a seemingly obvious relation

$$
\hat{Q}_{t r}=\int_{0}^{T} d t \hat{I}(t)
$$

Having this operator in hands, one applies the general paradigm of quantum measurement 1 : The probability to have a certain charge $q$ transferred equals the square of the projection of the wave function of the system on the eigenstate of $\hat{Q}_{t r}$ with eigenvalue $q$. Lesovik and Levitov were able to perform a challenging calculation of these projections. However, they were hardly satisfied with the results. For instance, the transferred charge was not quantized in units of the elementary charge.

This is why in the subsequent paper 3 the same authors have proposed another method of evaluating $P_{T}(N)$. Their scheme invoked an extra measuring device. As a model device, they chose a precessing $1 / 2$ spin whose precession angle should be proportional to the transferred charge. The measurement paradigm is then applied to the device. 
In this way they were able to obtain a satisfactory definition of the full counting statistics $P_{T}(N)$ with an integer number of charges transferred. The details of the calculation and a thorough discussion are presented in 1

It was clear to the authors of that their definition of the FCS does not depend on a specific measurement scheme. However, this fact was not explicitly evident. For several years this hindered the impact of these outstanding contributions.

One of the authors has recently proposed a slightly different calculation scheme of FCS that does not implicitly invoke any measuring device but still leads to the same results 1 . The observation was that the cumulants of the current can be obtained as non-linear responses of a system to a fictitious field that can only be defined in the framework of the Keldysh diagrammatic technique 6 . The calculation of FCS can be accomplished with a slight extension of the Keldysh technique. This meant some progress since the methods of the Keldysh technique are well elaborated and can be readily pplied to a variety of physical systems and situations.

Recent work $\mathrm{d}$ has addressed the charge transfer between two superconductors. The problem can be tackled with an extension of the above-mentioned Keldysh technique. The expressions for $P_{T}(N)$ were obtained. Albeit the authors have encountered a significant difficulty with understanding the results in classical terms. The point is that the calculation gave negative probabilities. This indicates that the results cannot be interpreted without invoking a quantum mechanical description of a detector.

All this suggests that the quantum mechanical concept of full counting statistics shall be refined and the generality of previously used definitions shall be accessed. This is done in the present article.

To preserve generality, we focus on the full counting statistics of an arbitrary quantum mechanical variable $A$. Then the result does not have to be discrete, and, strictly speaking, no counting takes place. We keep the term "counting" for historical reasons.

We show that introducing a detector is absolutely necessary for defining such statistics. On the other hand, the answer does not depend on details of the detector. This allows for a separation of the system measured and the measuring device. We develop an exact quantum mechanical description of the system in terms of a path integral over detector variables and derive our results from this description. We show that the classical interpretation of full counting statistics is only possible in the presence of a certain symmetry. For the full counting statistics of the electric current, this symmetry is gauge invariance. In superconductors the gauge invariance is broken and the full counting statistics must be interpreted along quantum mechanical lines.

It is the main message of our paper that this interpretation problem does not make the concept of full counting statistics useless and/or unphysical. We show that it is the full counting statistics that completely determines the evolution of the density matrix of the detector. We show that thereby the statistics is observable in real experiments. We propose and discuss two concrete measuring schemes.

The paper is organized as follows. We start with a general compact discussion of the interpretation problems. We present the model of the detector in section III. We present the approach and derive general relations for the detector density matrix in the subsequent section. Section $\mathrm{V}$ defines the FCS and presents its interpretation. The subsequent sections provide examples of FCS for an equilibrium system in the ground state, a normal conductor and a harmonic oscillator. The characterization of the FCS is considered in sections $\mathrm{IX}$ and $\mathrm{X}$ where two concrete measurement schemes are discussed.

\section{GENERAL DISCUSSION}

It is not a priori clear why the operator definition (1) produces senseless results. We list below possible intuitive reasons for this. To start with, the paradigm concerns an instant measurement. The operator definition (11) is not local in time and accumulates information about the quantum state of the system for a (long) interval of time. The applicability of the paradigm is therefore not obvious. For instance, the averages of powers of $\hat{Q}_{t r}$ can be expressed in terms of correlators of currents

$$
<\hat{Q}_{t r}^{N}>=\int_{0}^{T} d t_{1} \ldots d t_{N}<\hat{I}\left(t_{1}\right) \hat{I}\left(t_{2}\right) \ldots \hat{I}\left(t_{N}\right)>
$$

Usually the causality comes into quantum mechanics via time ordering of operator products. There is no time ordering of current operators in (2). This may indicate implicit problems with causality. The second reason is as follows. It seems obvious that the time integral of $\hat{I}$ can be associated with a physical operator of charge. For an arbitrary operator $\hat{A}$ it may be difficult to find such a physical associate. However, the integrals of $\hat{A}$ can be measured, and statistics of those can be accumulated.

The only way out seems to extend the original quantum mechanical system by adding a "detector". Although it is very physical, mathematically this means an eventual doubling of the original Hilbert space by adding extra 
degrees of freedom. In fact, this has been done in 3 by introducing the spin- $1 / 2$. Below we adopt the simplest way to provide such an extensions. Given a quantum mechanical system, one introduces an extra variable $x$, whose operator $\hat{x}$ commutes with all operators of the system. We assume that the canonically conjugated variable, $q$, $([\hat{x}, \hat{q}]=i$, in units with $\hbar=1)$ can be measured according to the paradigm. Next we introduce an interaction between the system and the detector in such a way that in the time interval $(0, T)$ the Heisenberg equation of motion becomes

$$
\dot{\hat{q}}(t)=\hat{A}(t)
$$

thus simulating Eq. (11). In this way we avoid all possible difficulties with misinterpretations of the paradigm. The integral of $A(t)$ is now correctly associated with an operator of a value that can be a priori measured.

Albeit there is a price to pay. As we show below, the FCS can be defined in this way as an operator that relates the density matrices of the detector before and after the measurement. In general, it is not the same as the probability distribution of the shifts of the detector momentum which may be defined in the classical limit. The FCS can be interpreted in such terms only under certain conditions, which are satisfied for the statistics of current in normal conductors. However, the definition we adopt is sufficiently constructive since it allows to predict the result of any realistic measurement.

To make the detector more realistic and thus to show the generality of the results, one shall introduce internal dynamics of the detector variable. This dynamics would make the detector a non-ideal one: the readings may differ from the definitions (11) and (3). The path integral approach we describe below provides the most convenient way to incorporate this internal dynamics.

\section{THE MODEL}

Let us now specify our detection model. The detector will formally be a particle with the Hamiltonian $\frac{\hat{q}^{2}}{2 m}+V(\hat{x})$. $\hat{q}$ and $\hat{x}$ obey canonical commutation relations. It shall be coupled to the system only for a certain period of time $T$ and be decoupled adiabatically for earlier and later times. We introduce a smooth coupling function $\alpha_{T}(t)$ that takes the value 1 in the time interval $0<t<T$ and is zero beyond the time interval $t_{1}<t<t_{2}$. The values for $t_{1}<0$ and $t_{2}>T$ are chosen in a way that provides an adiabatic switching. The whole Hamiltonian therefore reads

$$
\hat{H}(t)=\hat{H}_{\mathrm{sys}}-\alpha_{T}(t) \hat{x} \hat{A}+\frac{\hat{q}^{2}}{2 m}+V(\hat{x})
$$

We stress that this detector model is very general. It can easily be applied to describe a variety of electrical circuits and mechanical systems. In particular, it may describe selective detectors: by choosing $V(x)=\frac{m}{2} \omega^{2} x^{2}$ we describe a harmonic oscillator that selectively detects a signal frequency $\omega$. In principle it would also be possible to treat detectors that consist of more than one degree of freedom in the formalism we are going to develop.

The coupling is chosen such that the Heisenberg equation of motion for the detector quasimomentum $\hat{q}$

$$
\dot{\hat{q}}(t)=\alpha_{T}(t) \hat{A}(t)-V^{\prime}(\hat{x}(t))
$$

reproduces Eq. (3) if the internal dynamics of the detector is disregarded. Eq. (5) suggests that the statistics of measurements of the detector's momentum after uncoupling it from the system will tell us about the full counting statistics we are interested in. The coupling term can be viewed as a disturbance of the system measured by the measuring device. To minimize this disturbance, we would clearly like to concentrate the detector wave function around $x=0$. The uncertainty principle forbids us, however, to localize it completely. Indeed, in such a way we would loose all information about the momentum, which we are going to measure. This is a fundamental limitation imposed by quantum mechanics, and we are going to explore its consequences step by step. To concentrate fully on the quantum nature of the detector we explore in details the limit of infinite detector mass. In this limit, $\dot{\hat{x}}=0$ and the detector can classically not disturb the system.

\section{THE APPROACH}

Our goal is to relate the density matrices of the detector before and after the measurement. If there were no system to measure we could easily express this relation in the form of a path integral in the (double) variable $x(t)$, like it has been done many years ago in $\theta$. The expectation is that the whole information about the system to be measured can be compressed into an extra factor in this integral, the so-called influence functional. This functional will depend on 
$x(t)$ rather than on the internal dynamics of the detector. This will make explicit the separation between the detector and the measured system. To make contact with 1 , we would like to present this influence functional as an operator expression that involves the system degrees of freedom only. We denote the initial detector density matrix (at $t<t_{1}$ ) by $\rho^{i n}\left(x^{+}, x^{-}\right)$and the final one (at $t>t_{2}$, after having traced out the system's degrees of freedom) by $\rho^{f}\left(x^{+}, x^{-}\right)$. $\hat{R}$ is the initial unperturbed density matrix of the system. The entire initial density matrix is assumed to factorize, $\hat{D}=\hat{R} \hat{\rho}^{i n}$.

We start out by inserting complete sets of states into the expression for the time development of the density matrix

$$
\begin{gathered}
\hat{\rho}^{f}\left(x^{+}, x^{-}\right)=\underset{\text { System }}{\operatorname{Tr}} \\
<x^{+}\left|\vec{T} \exp \left\{-i \int_{t_{1}}^{t_{2}} d t\left[\hat{H}_{\mathrm{sys}}-\alpha_{T}(t) \hat{x} \hat{A}+\frac{\hat{q}^{2}}{2 m}+V(\hat{x})\right]\right\} \hat{D} \overleftarrow{T} \exp \left\{i \int_{t_{1}}^{t_{2}} d t\left[\hat{H}_{\mathrm{sys}}-\alpha_{T}(t) \hat{x} \hat{A}+\frac{\hat{q}^{2}}{2 m}+V(\hat{x})\right]\right\}\right| x^{-}>
\end{gathered}
$$

in the usual manner. $\vec{T}(\overleftarrow{T})$ denotes (inverse) time ordering. As the complete sets of states we choose product states of any complete set of states of the system and alternatingly complete sets of eigenstates of the position or the momentum operator of the detector. Those intermediate states allow us to replace the position and momentum operators in the time development exponentials by their eigenvalues. We can then do the integrals over the system states as well as the momentum integrals and arrive at the expression

$$
\begin{gathered}
\left.\hat{\rho^{f}}\left(x^{+}, x^{-}\right)=\int \begin{array}{cc}
D\left[x^{+}(t)\right] & D\left[x^{-}(t)\right] \\
x^{+}\left(t_{2}\right)=x^{+} & \exp \left\{i \int_{t_{1}}^{t_{2}} d t_{2}\right)=x^{-}
\end{array} d t \frac{m}{2}\left[\left(\dot{x}^{+}\right)^{2}-\left(\dot{x}^{-}\right)^{2}\right]+V\left(x^{+}(t)\right)-V\left(x^{-}(t)\right)\right\} \underset{\text { System }}{T r} \\
\vec{T} \exp \left\{-i \int_{t_{1}}^{t_{2}} d t\left[\hat{H}_{\mathrm{sys}}-\alpha_{T}(t) x^{+}(t) \hat{A}\right]\right\} \hat{R} \overleftarrow{T} \exp \left\{i \int_{t_{1}}^{t_{2}} d t\left[\hat{H}_{\mathrm{sys}}-\alpha_{T}(t) x^{-}(t) \hat{A}\right]\right\} \rho^{i n}\left(x^{+}\left(t_{1}\right), x^{-}\left(t_{1}\right)\right)
\end{gathered}
$$

We rewrite the expression as

$$
\rho^{f}\left(x^{+}, x^{-}\right)=\int d x_{1}^{+} d x_{1}^{-} K\left(x^{+}, x^{-} ; x_{1}^{+}, x_{1}^{-}, T\right) \rho^{i n}\left(x_{1}^{+}, x_{1}^{-}\right)
$$

in terms of the kernel

$$
\begin{aligned}
& K\left(x^{+}, x^{-} ; x_{1}^{+}, x_{1}^{-}, T\right)=\int \begin{array}{cc}
D\left[x^{+}(t)\right] & D\left[x^{-}(t)\right] \\
x^{+}\left(t_{2}\right)=x^{+}, x^{+}\left(t_{1}\right)=x_{1}^{+} & x^{-}\left(t_{2}\right)=x^{-}, x^{-}\left(t_{1}\right)=x_{1}^{-}
\end{array} \\
& F\left[x^{+}(t), x^{-}(t), T\right] \exp \left\{i \int_{t_{1}}^{t_{2}} d t \frac{m}{2}\left[\dot{x}^{+}(t)^{2}-\dot{x}^{-}(t)^{2}\right]+V\left(x^{+}(t)\right)-V\left(x^{-}(t)\right)\right\}
\end{aligned}
$$

that contains already the desired influence functional

$$
F\left[x^{+}(t), x^{-}(t), T\right]=\underset{\text { System }}{\operatorname{Tr}} \vec{T} \exp \left\{-i \int_{t_{1}}^{t_{2}} d t\left[\hat{H}_{\mathrm{sys}}-\alpha_{T}(t) x^{+}(t) \hat{A}\right]\right\} \hat{R} \overleftarrow{T} \exp \left\{i \int_{t_{1}}^{t_{2}} d t\left[\hat{H}_{\mathrm{sys}}-\alpha_{T}(t) x^{-}(t) \hat{A}\right]\right\}
$$

We have therefore accomplished the task: system and detector are presented as separate terms under the sign of path integration.

Seeking further simplifications, we proceed by taking the limit of infinite detector mass and considering a free particle, so that $V(x)=0$. We find that the kinetic term in Eq. (9) then suppresses all fluctuations in the path integral. The details of the calculation needed to establish this are provided in Appendix A. Therefore, the kernel $K\left(x^{+}, x^{-}, x_{1}^{+}, x_{1}^{-}, T\right)$ becomes local in position space, does not depend on the detector and reads

$$
K\left(x^{+}, x^{-}, x_{1}^{+}, x_{1}^{-}, T\right)=\delta\left(x^{+}-x_{1}^{+}\right) \delta\left(x^{-}-x_{1}^{-}\right) P\left(x^{+}, x^{-}, T\right)
$$

with

$$
P\left(x^{+}, x^{-}, T\right)=\underset{\text { System }}{T r} \vec{T} \exp \left\{-i \int_{t_{1}}^{t_{2}} d t\left[\hat{H}_{\mathrm{sys}}-\alpha_{T}(t) x^{+} \hat{A}\right]\right\} \hat{R} \overleftarrow{T} \exp \left\{i \int_{t_{1}}^{t_{2}} d t\left[\hat{H}_{\mathrm{sys}}-\alpha_{T}(t) x^{-} \hat{A}\right]\right\}
$$

If we compare expressions (10) and (12) we see that the simplification is that the $x^{ \pm}(t)$ do not depend on time. So the detector is somehow "slow" and the variable $x(t)$ quasi-stationary. This gives physical sense to the limit we consider. The only requirement is eventually time scale separation between the system and the detector. The typical time scale 
of the internal dynamics of the detector shall exceed that one of the system. Under these conditions, we can apply the quasi-stationary approximation and replace $F$ with $P$.

It is constructive to rewrite now the density matrices in Wigner representation

$$
\rho(x, q)=\int \frac{d z}{2 \pi} e^{i q z} \rho\left(x+\frac{z}{2}, x-\frac{z}{2}\right)
$$

and define

$$
P(x, q, T)=\int \frac{d z}{2 \pi} e^{i q z} P\left(x+\frac{z}{2}, x-\frac{z}{2}, T\right) .
$$

This gives the following convenient relation

$$
\rho^{f}(x, q)=\int d q_{1} P\left(x, q-q_{1}, T\right) \rho^{i n}\left(x, q_{1}\right)
$$

\section{INTERPRETATION OF FCS}

We adopt the relations (12), (14) and (15) as the definition of the FCS of the variable $A$. Let us see why. First let us suppose that we can treat the detector classically. Then the density matrix of the detector in Wigner representation can be interpreted as a classical probability distribution $\Pi(x, q)$ to be at a certain position $x$ with momentum $q$. This allows for a classical interpretation of $P(x, q, T)$ as a probability to have measured $q=\int_{0}^{T} A(t)$. Indeed, one sees from (14) that the final $\Pi(x, q)$ is obtained from the initial one by shifts in $q, P(x, q, T)$ being the probability distribution of those shifts.

Albeit there is a point which hampers such classical interpretation. The density matrix in Wigner representation cannot be interpreted as a probability to have a certain position and momentum or any other probability, since it is not positive. Concrete calculations given below illustrate that $P(x, q, T)$ does not have to be positive as well. Consequently, it cannot be interpreted as a probability distribution. We stress that the absence of a classical interpretation does not hamper a "predictive power" of FCS: having it in hands, one can predict the results of measurements using Eq. (12). The reverse problem is to extract the FCS from the results of measurements. We discuss two schemes for this in sections IX and $\mathrm{X}$.

There is an important case where FCS can indeed be interpreted as a probability distribution. In this case $P(x, q, T)$ does not depend on $x, P(x, q, T) \equiv P(q, T)$. Then, integrating Eq. (15) over $x$, we find

$$
\Pi^{f}(q)=\int d q^{\prime} P\left(q-q^{\prime}, T\right) \Pi^{i n}\left(q^{\prime}\right)
$$

with $\Pi(q) \equiv \int d x \rho(x, q)$. Therefore, the FCS is in this special case the kernel that relates the probability distributions of the detector momentum before and after the measurement, $\Pi^{i n}(q)$ and $\Pi^{f}(q)$, to each other. Those distributions are positive and so will the FCS $P(q, T)$. Note, that a generic case in which this interpretation is appropriate is the measurement of a static operator $\hat{A}$, that is, $\left[\hat{H}_{\text {sys }}, \hat{A}\right]=0$. Then the FCS is obviously $x$-independent and it can be interpreted as the probability distribution of outcomes of an instant measurement of $\hat{A}$.

Since we study FCS of a stationary system and the measurement time $T$ exceeds timescales associated with the system, the operator expression in Eq. (12) can be seen as a product of terms corresponding to time intervals. Therefore in this limit of $T \rightarrow \infty$ the dependence on the measuring time can be reconciled into

$$
P\left(x^{+}, x^{-}\right)=e^{-\mathcal{E}\left(x^{+}, x^{-}\right) T}
$$

where the expression in the exponent is supposed to be big. Then the integral (14) that defines FCS can be done in the saddle point approximation. If one defines $A=q / T$, that is, $A$ is the result of measurement of $\int A(t) d t / T$, the FCS can be recast into the similar from

$$
P(x, A, T)=e^{-\tilde{\mathcal{E}}(x, A) T}
$$

where $\tilde{\mathcal{E}}$ is defined as a (complex) extremum with respect to (complex) $z$ :

$$
\tilde{\mathcal{E}}=\operatorname{extr}_{z}\left\{\mathcal{E}\left(x+\frac{z}{2}, x-\frac{z}{2}\right)-i A z\right\}
$$


The average value of $A$ and its variance (noise) can be expressed in terms of derivatives of $\mathcal{E}$ :

$$
<A>=\lim _{z \rightarrow 0} \frac{\partial \mathcal{E}(x+z / 2, x-z / 2)}{i \partial z} ; \quad T \ll A^{2} \gg=\lim _{z \rightarrow 0} \frac{\partial^{2} \mathcal{E}(x+z / 2, x-z / 2)}{\partial z^{2}} .
$$

Indeed, the quantity $P\left(x^{+}, x^{-}, T\right)$ is the generating function of moments of $q$. It is interesting to note that in general this function may generate a variety of moments that differ in the time order of operators involved, for instance,

$$
Q_{M}^{N}=(-i)^{N} \lim _{x^{ \pm} \rightarrow 0} \frac{\partial^{M}}{\partial\left(x^{-}\right)^{M}} \frac{\partial^{N-M}}{\partial\left(x^{+}\right)^{N-M}} P\left(x^{+}, x^{-}\right)=\int_{0}^{T} d t_{1} \ldots d t_{N}<\overleftarrow{T}\left\{\hat{A}\left(t_{1}\right) \ldots \hat{A}\left(t_{M}\right)\right\} \vec{T}\left\{\hat{A}\left(t_{M+1}\right) \ldots \hat{A}\left(t_{N}\right)\right\}>
$$

The moments of $q$ are expressed through these moments and binomial coefficients

$$
Q^{(N)} \equiv \int d q q^{N} P(0, q, T)=2^{-N} \sum_{M} C_{M}^{N} Q_{M}^{N}
$$

\section{FCS OF AN EQUILIBRIUM SYSTEM IN THE GROUND STATE}

To acquire a better understanding of the general relations obtained we consider now an important special case. We will assume that the system considered is in its ground state $|g\rangle$, so that its initial density matrix is $\hat{R}=|g\rangle\langle g|$. This assumption allows for an easy calculation of the FCS. We have supposed that the coupling between the system and the detector arises adiabatically. Then the time development operators in (12) during the time interval $t_{1}<t<0$ adiabatically transfer the system from $\mid g>$ into the ground state $\mid g\left(x^{ \pm}\right)>$of the new Hamiltonian $\hat{H}_{\text {sys }}-x^{ \pm} \hat{A}$. In the time interval $0<t<T$ the time evolution of the resulting state has thus the simple form

$$
\exp \left\{-i t\left(\hat{H}_{\mathrm{sys}}+x^{ \pm} \hat{A}\right)\right\}\left|g\left(x^{ \pm}\right)>=e^{-i t E\left(x^{ \pm}\right)}\right| g\left(x^{ \pm}\right)>.
$$

$E\left(x^{ \pm}\right)$are the energies corresponding to $\mid g\left(x^{ \pm}\right)>$. This provides the main contribution to the FCS if the measurement time is large and the phase acquired during the switching of the interaction can be neglected in comparison with this contribution. Therefore we obtain

$$
P\left(x^{+}, x^{-}, T\right)=e^{-i T\left(E\left(x^{+}\right)-E\left(x^{-}\right)\right)} .
$$

First we observe that $P(x, q, T)$ is a real function in this case, since the exponent in 25) is anti-symmetric in $z$. A first requirement for being able to interpret $P(0, q, T)$ as a probability distribution is therefore fulfilled. However, the same asymmetry assures that all even cumulative moments of $q$ are identically zero, whereas the odd ones need not. On one hand, since the second moment corresponds to the noise and the ground state cannot provide any, this makes sense. On the other hand, this situation would be impossible if $P(0, q, T)$ were a true, positive probability distribution.

Belzig and Nazarov encountered this situation analysing the FCS of a general super-conducting junction. In a certain limit the junction becomes a simple Josephson junction in its ground state. In this mere limit the interpretation of FCS as a probability distribution does not work any longer. Fortunately enough, the relation (15) allows us to interpret the results obtained.

To keep simple things simple, we assume the function $E(x)$ to be analytic and expand it in its Taylor series. We also rescale $q$ as above, $q=A / T$. For the FCS we therefore have

$$
P(x, A, T)=\int \frac{d z}{T} e^{i z A T} \cdot e^{-i T\left(E^{\prime}(x) z+E^{\prime \prime \prime}(x) z^{3} / 24+\ldots\right)}
$$

If we take the limit $T \rightarrow \infty$ now, we note that all the terms involving higher derivatives of $E(x)$ are negligible and arrive at

$$
\lim _{T \rightarrow \infty} P(x, A, T)=\delta\left(A-E^{\prime}(x)\right)
$$

According to the Hellman-Feynman theorem $E^{\prime}(x)=<g(x)|\hat{A}| g(x)>$. As one would expect, in this limit the measurement gives the expectation value of the operator $\hat{A}$ in a ground state of the system that is somewhat altered by its interaction with the detector at position $x$. Therefore the resulting dispersion of $A$ will be determined by the initial quantum mechanical spread of the detector wave function. Consequently, the error of the measurement comes from the interaction with the detector rather than from the intrinsic noise of the system measured. 


\section{FCS OF THE ELECTRIC CURRENT IN A NORMAL CONDUCTOR}

A complementary example is a normal conductor biased at finite voltage. This is a stationary non-equilibrium system far from being in its ground state. Here we do not intend to go to microscopic details of the derivation. Our immediate aim is to make contact with the approaches of Refs. 4 - We keep the original notations of the references wherever it is possible.

The starting points of the approaches differ much. Levitov and Lesovik propose a detector model where the $z$ component of a spin- $1 / 2$ creates a local vector potential which is felt by the electrons. This corresponds to a total Hamiltonian of the form

$$
\hat{H}=\hat{H}_{\mathrm{sys}}-\frac{\lambda}{2 e} \hat{\sigma}_{z} \hat{I}
$$

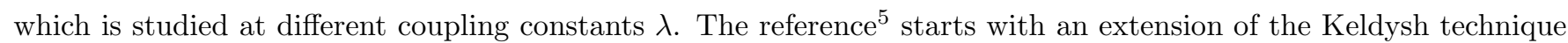
to formally unphysical systems where the evolution of the wave function in different time directions is governed by two different Hamiltonians

$$
\hat{H}^{ \pm}=\hat{H}_{\text {sys }} \pm \chi \hat{I}
$$

and shows that the so defined Green functions can be used to generate moments of $\hat{I}$. This is to be compared with our detection model.

Despite different starting points, all three approaches quickly concentrate on the calculation of the quantity

$$
<\exp \left(-i \hat{H}_{1} T\right) \exp \left(i \hat{H}_{2} T\right)>
$$

which is identical to our definition of $P\left(x^{+}, x^{-}\right)$provided that $H_{1,2}=\hat{H}_{\text {sys }}-x^{ \pm} \hat{I}$. This quantity is denoted by $\chi(\lambda)$ in 1 and by $\exp (-S(\chi))$ in 5 . It is used to define the FCS and we see now that the final result does not depend on the starting point.

To give a concrete expression for FCS, we just make use of Eq. (37) ofl . We consider the FCS of the current in a multi-mode constriction which is characterized by a set of transmission coefficients $T_{n}$. In general, the answer is expressed in terms of energy-dependent electron filling factors $n_{R(L)}$ on the right (left) side of the constriction

$$
P\left(x^{+}, x^{-}, T\right)=\exp \left\{\frac{T}{2 \pi} \sum_{n} \int_{-\infty}^{+\infty} d E \ln \left(1+T_{n}\left(e^{i\left(x^{-}-x^{+}\right) / e}-1\right) n_{R}\left(1-n_{L}\right)+T_{n}\left(e^{i\left(x^{+}-x^{-}\right) / e}-1\right) n_{L}\left(1-n_{R}\right)\right)\right\}
$$

We stress that this expression depends on $x^{+}-x^{-}$only. This is a direct consequence of gauge invariance. Indeed, in each of the Hamiltonians the coupling term is the coupling to a vector potential localized in a certain cross-section of the constriction. The gauge transform that shifts the phase of the wave functions by $x^{ \pm}$fo on the right side of the constriction, eliminates this coupling term. This transform was explicitly implemented in 5 . Since there are two Hamiltonians in the expression, the coupling terms cannot be eliminated simultaneously provided $x^{+} \neq x^{-}$. However, the gauge transform with the phase shift $\left(x^{+}+x^{-}\right) / 2$ makes the coupling terms depending on $x^{+}-x^{-}$only.

Since $P\left(x^{+}, x^{-}, T\right)$ depends on $x^{+}-x^{-}$only, the $\operatorname{FCS} P(x, q, T)$ does not depend on $x$. As we have stressed in section $\mathrm{V}$, this enables one to interpret the FCS as a probability distribution.

Superconductivity breaks gauge invariance, thus making such an interpretation impossible.

\section{FCS OF A HARMONIC OSCILLATOR}

Let us now illustrate the measuring process proposed with a simple example. We consider the measurement of the position of a harmonic oscillator in its ground state. That is, $\hat{H}_{0}=\frac{\hat{Q}^{2}}{2 M}+\frac{1}{2} M \omega^{2} \hat{X}^{2}$ is the Hamiltonian of the system and $\hat{A}=\hat{X}$ shall be measured. The measurement is effected by coupling the oscillator to a detector with position variable $x$ during a time interval of length $T$. In the limit of infinite detector mass the entire Hamiltonian in the measurement period reads then

$$
\hat{H}=\frac{\hat{Q}^{2}}{2 M}+\frac{1}{2} M \omega^{2} \hat{X}^{2}+\hat{x} \hat{X}
$$

The perturbed ground state $\mid g(x)>$ is in this simple example obtained by shifting the original ground state wave function by $x / M \omega^{2}$ in $X$-representation. Its energy is $E_{g}(x)=E_{g}(0)-\frac{1}{2 M \omega^{2}} x^{2}$. We then find from (25), that 


$$
P(x, q, T)=\delta\left(q+x T / M \omega^{2}\right)
$$

Following our first classical interpretation of $P(0, q, T)$ we would now conclude, that a harmonic oscillator in its ground state does not transmit any fluctuations of its position variable to the detector and that the detector's wave function is not altered by the oscillator. Let us, however, calculate the read off of the detector after the measurement just described, i.e., its final momentum distribution. We have to integrate the final density matrix found from (15) over $x$. As the initial detector state we choose a Gaussian wave with uncertainty $\triangle q$ in the momentum:

$$
\rho^{i n}(x, q)=\exp \left[-\frac{q^{2}}{2(\triangle q)^{2}}-2(\triangle q)^{2} x^{2}\right]
$$

The final momentum distribution

$$
\Pi^{f}(q)=\exp \left[\frac{-q^{2}}{2(\triangle q)^{2}+T^{2} / 2 M^{2} \omega^{4}(\triangle q)^{2}}\right]
$$

is again a Gaussian. Its width $\triangle q^{f}$, however, increases in time, for big times linearly with $T$ :

$$
\left(\triangle q^{f}\right)^{2}=(\triangle q)^{2}+\frac{T^{2}}{4 M^{2} \omega^{4}(\triangle q)^{2}}
$$

This relation clearly demonstrates the relevance of the detector's influence on the system due to its quantum nature. The initial detector momentum is known only with an uncertainty $\triangle q$. This uncertainty is a source of measurement error and is described by the first term of Eq. (34). We would clearly like to minimize this error by choosing $\triangle q$ to be small. Doing so, we increase, however, the uncertainty in the detector position $\triangle x \gtrsim 1 / 2 \triangle q$. According to Eq. (30), such a spread in the detector position will disturb the system. Since the oscillator is in its ground state this disturbance will drive it into ground states of new Hamiltonians $\hat{H}_{0}+x \hat{X}$. For every detector influence $x$ we will therefore measure a different expectation value $E^{\prime}(x)=<g(x)|\hat{X}| g(x)>$. The read off of the detector will be a superposition of measurement outcomes corresponding to all those different influences. The resulting broadening of the final detector momentum distribution $\left(\triangle q^{f}\right)_{\triangle x} \approx \triangle x E^{\prime \prime}(0) T$ is accounted for by the second term in (34). We conclude that the the quantum fluctuations of the detector set an upper bound on the accuracy of the measurement process. The actual value of this bound depends of course on the system measured. It will vanish if the FCS is $x$-independent and a classical interpretation of the process is appropriate.

We have seen that the FCS is at least not directly observable in a single experiment. It is buried under some additional broadening which we could fully understand in terms of the disturbance of the system by the detector. It therefore does not tell us anything about fluctuations of the observable $A$ in our system, the quantity we want to measure after all. In the next section we will therefore try to get rid of this broadening and measure the function $P(x, q, T)$ itself, which is more specific to the system. A certain deconvolution procedure will be developed.

\section{CHARACTERIZATION OF THE FCS; FIRST SCHEME}

As we have already seen, the full counting statistics $P(x, q, T)$ proposed above allows us to predict the outcomes of measurements with a quite general detector and resolves inconsistencies that arose in earlier interpretations. It remains to show now, that it is a physical, real object in the sense that it is experimentally observable.

We would like to suggest two schemes for measuring the FCS. We start from relation (15) between the initial and the final density matrix. Writing this equation in $\left(x^{+}, x^{-}\right)$-space, we find the simple expression

$$
P\left(x^{+}, x^{-}, T\right)=\frac{\rho_{T}^{f}\left(x^{+}, x^{-}\right)}{\rho^{i n}\left(x^{+}, x^{-}\right)}
$$

or

$$
P(x, q, T)=\int \frac{d z}{2 \pi} e^{i q z} \frac{\rho_{T}^{f}(x+z / 2, x-z / 2)}{\rho^{i n}(x+z / 2, x-z / 2)}
$$

for the kernel. We would already be done if we could measure all elements of the detector's final and initial density matrices. This is not possible in general, however. By successively measuring a certain observable we can measure the diagonal elements of the density matrix in a basis of eigenstates of that observable, but not the off-diagonal entries. We can therefore measure the functions $\Pi(q)$, but not $\hat{\rho}$ itself. 
The key idea that we will pursue to solve this problem is to repeat the same measurement many times for shifted but otherwise identical initial detector density matrices. We suggest to repeat the measurement of the final momentum distribution $\Pi^{f}(q)$ for a number of initial density matrices that differ only in the expectation value $x_{0}$ of the position of the detecting particle and call it

$$
\Gamma^{f}\left(x_{0}, q, T\right) \equiv \Pi_{T}^{f}\left(q, x_{0}\right)=\int d x d q^{\prime} P\left(x, q-q^{\prime}, T\right) \rho^{i n}\left(x-x_{0}, q^{\prime}\right) .
$$

This way we expose the system during the measurement to different detector influences and one can hope that by doing so this influence can be identified and eliminated by some kind of a deconvolution procedure. Defining the Fourier transform of $\Gamma^{f}\left(x_{0}, q, T\right)$ with respect to both of its variables

$$
\tilde{\Gamma}^{f}\left(q_{0}, z, T\right) \equiv \frac{1}{2 \pi} \int d x_{0} d q e^{i x_{0} q_{0}-i z q} \Gamma^{f}\left(x_{0}, q, T\right)
$$

we find, that the FCS can indeed be reconstructed from this function by means of the relation

$$
P(x, q, T)=\frac{1}{2 \pi} \int d q_{0} d z e^{i q z-i q_{0} x} \frac{\tilde{\Gamma}^{f}\left(q_{0}, z, T\right)}{\tilde{\rho}^{i n}\left(q_{0}, z\right)}
$$

where

$$
\tilde{\rho}\left(q_{0}, z\right) \equiv \int d x e^{i q_{0} x} \rho\left(x+\frac{z}{2}, x-\frac{z}{2}\right)
$$

To interpret the result of the measurement, we still have to know the full initial density matrix of the detector. This should be feasible, however. One might either prepare the detector initially in a specific, well-known state, or one might let the detector equilibrate with an environment. Then the initial density matrix is stationary, $0=\left[\hat{\rho}^{i n}, \hat{H}\right] \propto\left[\hat{\rho}^{i n}, \hat{q}^{2}\right]$, it will be diagonal in a basis of momentum eigenstates and can be determined by a momentum measurement only. This proves that the FCS really is an observable.

To illustrate the procedure we want to apply it now to our example of the harmonic oscillator already introduced earlier. Like there $\Pi^{f}(q)$ we calculate now the function $\Gamma^{f}\left(x_{0}, q, T\right)$ that would be measured in the actual experiment. We shift the initial oscillator wavefunction by $x_{0}$ in $x$-space and find that

$$
\Gamma^{f}\left(x_{0}, q, T\right)=\exp \left[\frac{-\left(q+x_{0} T / M \omega^{2}\right)^{2}}{\left(2(\triangle q)^{2}+T^{2} / 2 M^{2} \omega^{4}(\triangle q)^{2}\right)}\right] .
$$

On transforming this into Fourier space it becomes

$$
\tilde{\Gamma}^{f}\left(q_{0}, z, T\right)=\exp \left[-\frac{(\triangle q)^{2} z^{2}}{2}-\frac{T^{2} z^{2}}{8 M^{2} \omega^{4}(\triangle q)^{2}}\right] \delta\left(q_{0}+T z / M \omega^{2}\right) .
$$

Employing now Eq. (39) with $\rho^{\tilde{i}}\left(q_{0}, z\right)=\exp \left[-\frac{(\triangle q)^{2} z^{2}}{2}-\frac{q_{0}^{2}}{8(\triangle q)^{2}}\right]$ we indeed recover the desired FCS Eq. (31).

\section{SECOND SCHEME}

We see also a possibility of measuring $P(x, q, T)$ without knowledge of the initial state of the detector. We can apply it, however, only to the special case in which the system is in one state only, for example its ground state, or in a mixture of a limited number of discrete states. This condition may be satisfied at low temperatures or if we have dissipation in the system which drives it into the ground state. We explain the idea for the case of a system in its ground state. Then we have the explicit expression (24) for the time evolution and we find, that

$$
\Gamma^{f}\left(x_{0}, q, T\right)=\int d x d z d q^{\prime} e^{-i z\left(q-q^{\prime}\right)-i T\left(E^{\prime}(x) z+\frac{1}{24} E^{\prime \prime \prime}(x) z^{3}+\ldots\right)} \rho^{i n}\left(x-x_{0}, q^{\prime}\right)
$$

$\Gamma^{f}\left(x_{0}, q, T\right)$ again being the final momentum distribution for shifted initial detector wave functions. In the limit of big $T$ we find with 26

$$
\lim _{T \rightarrow \infty} \Gamma^{f}\left(x_{0}, q, T\right) \propto \int d x \rho^{i n}\left(x-x_{0}, q-T E^{\prime}(x)\right) .
$$


This formula suggests that we can measure the function $E^{\prime}(x)$ arbitrarily exact in the limit of long measurement time $T$ by determining the peak of the final momentum distribution. The only assumption we have to make about the initial detector density matrix now is, that it is well centered around $x=0$ and that it falls off sufficiently fast for momenta higher than some arbitrary $\triangle q$. We want $\hat{\rho}^{\text {in }}$ to be peaked in $x$-space such that $E^{\prime}(x)$ is measured at the point $x_{0}$ only (we assume $\mathrm{E}(\mathrm{x})$ to be analytic). Of course, this means, that the width $\triangle q$ in momentum space of $\hat{\rho}^{i n}$ and therefore also of $\hat{\rho}_{T}^{f}$ will be rather wide. This is not a problem, however, since the position of the peak that we can measure with uncertainty $\triangle q$ increases linearly in time. The uncertainty in our knowledge of $E^{\prime}(x)$ is therefore determined by $\frac{\Delta q}{T}$ and tends to zero in the infinite time limit.

Integrating $E^{\prime}(x)$ we can then reconstruct the FCS for arbitrary detection times

$$
P(x, q, T)=\int \frac{d z}{2 \pi} \exp \left\{i q z-i T \int_{x-z / 2}^{x+z / 2} d x^{\prime} E^{\prime}\left(x^{\prime}\right)\right\}
$$

If the system is in a mixture of $N$ distinct states, the expression for $P(x, q, T)$ will be a sum of terms of the form (24) with different functions $E_{n}(x)$. We will in general find $N$ distinct peaks in the final momentum distribution allowing us to measure all $N$ functions $E_{n}^{\prime}(x)$. Again, we can reconstruct $P(x, q, T)$ for arbitrary $T$.

\section{CONCLUSIONS}

In this article we have been studying the process of measuring a general quantum mechanical variable $A$ by means of a detector that is coupled to it for a certain period of time. We have been focusing on a simple model describing a detector without internal dynamics which is nevertheless applicable to a variety of experimental situations. Moreover, the formalism that we have presented is general enough to allow for the description of a generic detector. We have predicted the outcome of such a measurement. This prediction involves a new object that we want to call full counting statistics (FCS) of the variable $A$ and that is an extension of another function proposed earlier in this context. This extension basically consists of accounting for the detector influence on the measured system. We find that the interplay of this influence with the quantum nature of the detector hampers in general a classical interpretation of the detector read-off. This way we have been able to remove inconsistencies, to be more specific, negative probabilities, that arose in the previous interpretation. Finally, we have shown, that this FCS is not only a theoretical construct that helps to predict results of certain measurements, but that it is an observable itself. It can be measured in experiments.

\section{ACKNOWLEDGMENTS}

We have benefitted from discussions with C.W.J. Beenakker, W. Belzig, and L.S. Levitov. This work was supported by the Dutch Science Foundation NWO/FOM.

\section{APPENDIX A:}

In this appendix we give a detailed derivation of the infinite mass limit of the kernel (9).

First, we define a Fourier transformed influence functional

$$
\begin{array}{r}
\tilde{F}\left[k^{+}(t), k^{-}(t), T\right]=\int D\left[x^{+}(t)\right] D\left[x^{-}(t)\right] F\left[x^{+}(t), x^{-}(t), T\right] \\
\exp \left\{i \int_{t_{1}}^{t_{2}} d t\left[x^{+}(t) k^{+}(t)-x^{-}(t) k^{-}(t)\right]\right\}
\end{array}
$$

and correspondingly

$$
\tilde{K}\left(q^{+}, q^{-}, q_{1}^{+}, q_{1}^{-}, T\right)=\int d x^{+} d x^{-} d x_{1}^{+} d x_{1}^{-} \exp \left\{i x^{+} q^{+}-i x^{-} q^{-}-i x_{1}^{+} q_{1}^{+}+i x_{1}^{-} q_{1}^{-}\right\} K\left(x^{+}, x^{-}, x_{1}^{+}, x_{1}^{-}, T\right)
$$

The $k^{ \pm}(t)$ are general functions on the interval $\left[t_{1}, t_{2}\right]$. Inserting the identity 


$$
\exp \left\{i \int_{t_{1}}^{t_{2}} d t \frac{m}{2} \dot{x}(t)^{2}\right\}=\int D[q(t)] \exp \left\{i \int_{t_{1}}^{t_{2}} d t\left[-\frac{q(t)^{2}}{2 m}-q(t) \dot{x}(t)\right]\right\}
$$

and using (9) we derive then

$$
\begin{array}{r}
\tilde{K}\left(q^{+}, q^{-}, q_{1}^{+}, q_{1}^{-}, T\right)=\int_{\substack{D \\
q^{+}\left(t_{2}\right)=q^{+}, q^{+}\left(t_{1}\right)=q_{1}^{+}}} \begin{array}{c}
D\left[q^{-}(t)\right] \\
q^{-}\left(t_{2}\right)=q^{-}, q^{-}\left(t_{1}\right)=q_{1}^{-}
\end{array} \\
\tilde{F}\left[\dot{q}^{+}(t), \dot{q}^{-}(t), T\right] \exp \left\{i \int_{t_{1}}^{t_{2}} d t\left(\frac{q^{+}(t)^{2}}{2 m}-\frac{q^{-}(t)^{2}}{2 m}\right)\right\}
\end{array}
$$

In the infinite mass limit, the kinetic term in this expression will disappear. Now, we change integration variables from $D\left[q^{ \pm}\right]$to $D\left[\dot{q}^{ \pm}\right]$and call $k^{ \pm}=\dot{q}^{ \pm}$. Then,

$$
\tilde{K}\left(q^{+}, q^{-}, q_{1}^{+}, q_{1}^{-}, T\right)=\int \underset{\int_{t_{1}}^{t_{2}} d t k^{+}(t)=q^{+}-q_{1}^{+}}{D\left[k_{t_{1}}^{t_{2}} d t k^{-}(t)=q^{-}-q_{1}^{-}\right.} \quad \stackrel{D\left[k^{-}(t)\right]}{\tilde{F}}\left[k^{+}(t), k^{-}(t), T\right]
$$

We can represent the functions $k^{ \pm}(t)$ by their Fourier series, $k^{ \pm}(t)=\sum_{n=0}^{\infty} k_{n}^{ \pm} \cos \frac{n \pi\left(t-t_{1}\right)}{t_{2}-t_{1}}$. Changing the integration variables in $A 5$ to the coefficients $k_{n}^{ \pm}$in this expansion, we notice, that only the integrals over the zeroth components $k_{0}^{ \pm}$are constrained by the boundary conditions. We can therefore do all the integrals over higher Fourier modes in (9) and obtain

$$
\begin{array}{r}
\tilde{K}\left(q^{+}, q^{-}, q_{1}^{+}, q_{1}^{-}, T\right)=\int \underset{\substack{D \\
n \neq 0}}{\substack {\left.k_{n}^{+}\right] \\
\begin{subarray}{c}{n \neq 0 \\
n \neq 0{ k _ { n } ^ { + } ] \\
\begin{subarray} { c } { n \neq 0 \\
n \neq 0 } }\end{subarray}}\left[k_{n}^{-}\right] D\left[x_{n}^{+}\right] D\left[x_{n}^{-}\right] \\
F\left[x^{+}(t), x^{-}(t), T\right] \exp \left\{\frac{i\left(t_{2}-t_{1}\right)}{2} \sum_{n=1}^{\infty}\left(k_{n}^{+} x_{n}^{+}-k_{n}^{-} x_{n}^{-}\right)+i\left[x_{0}^{+}\left(q^{+}-q_{1}^{+}\right)-x_{0}^{-}\left(q^{-}-q_{1}^{-}\right)\right]\right\}
\end{array}
$$

We have also expanded the functions $x^{ \pm}(t)$ in Fourier series now, $x^{ \pm}(t)=\sum_{n=0}^{\infty} x_{n}^{ \pm} \cos \frac{n \pi\left(t-t_{1}\right)}{t_{2}-t_{1}}$.

We see, that the $k_{n}^{ \pm}$-integrations result in $\delta$-functions that constrain the $x_{n}^{ \pm}, n \neq 0$, to zero and allow us to do the corresponding $x_{n}^{ \pm}$- integrals:

$$
\begin{array}{r}
\tilde{K}\left(q^{+}, q^{-}, q_{1}^{+}, q_{1}^{-}, T\right)=\int d x_{0}^{+} d x_{0}^{-} \exp \left\{i\left[x_{0}^{+}\left(q^{+}-q_{1}^{+}\right)-x_{0}^{-}\left(q^{-}-q_{1}^{-}\right)\right]\right\} \\
\underset{\text { System }}{\operatorname{Tr}} T \exp \left\{-i \int_{t_{1}}^{t_{2}} d t\left[\hat{H}_{\mathrm{sys}}-\alpha_{T}(t) x_{0}^{+} \hat{A}\right]\right\} \hat{R} \bar{T} \exp \left\{i \int_{t_{1}}^{t_{2}} d t\left[\hat{H}_{\mathrm{sys}}-\alpha_{T}(t) x_{0}^{-} \hat{A}\right]\right\}
\end{array}
$$

or

$$
K\left(x^{+}, x^{-}, x_{1}^{+}, x_{1}^{-}, T\right)=\delta\left(x^{+}-x_{1}^{+}\right) \delta\left(x^{-}-x_{1}^{-}\right) P\left(x^{+}, x^{-}, T\right)
$$

with

$$
P\left(x^{+}, x^{-}, T\right)=\underset{\text { System }}{\operatorname{Tr}} T \exp \left\{-i \int_{t_{1}}^{t_{2}} d t\left[\hat{H}_{\mathrm{sys}}-\alpha_{T}(t) x^{+} \hat{A}\right]\right\} \hat{R} \bar{T} \exp \left\{i \int_{t_{1}}^{t_{2}} d t\left[\hat{H}_{\mathrm{sys}}-\alpha_{T}(t) x^{-} \hat{A}\right]\right\} .
$$

Thus, the claimed locality of the kernel $K\left(x^{+}, x^{-}, x_{1}^{+}, x_{1}^{-}, T\right)$ is established.

${ }^{1}$ D. J. Griffiths, Introduction to Quantum Mechanics, Prentice Hall, New Jersey, 1995.

${ }^{2}$ L. S. Levitov and G. B. Lesovik, JETP Lett. 55, 555 (1992).

${ }^{3}$ L. S. Levitov and G. B. Lesovik, JETP Lett. 58, 230 (1993).

${ }^{4}$ L. S. Levitov, H. W. Lee, and G. B. Lesovik, Journ. Math. Phys. 37, 4845 (1996).

${ }^{5}$ Yu. V. Nazarov, Ann. Phys. (Leipzig) 8 Spec. Issue SI-193, 507 (1999).

${ }^{6}$ J. Rammer and H. Smith, Rev. Mod. Phys. 58, 323 (1986).

${ }^{7}$ W. Belzig and Yu. V. Nazarov, cond-mat/0012112.

${ }^{8}$ This has emerged from discussions with L. S. Levitov.

${ }^{9}$ R.P. Feynman and F.L. Vernon, Ann. Phys.(N.Y.) 24, 118 (1963). 